\title{
CONCENTRAÇÕES DE CLORO, SÓDIO, POTÁSSIO E FÓSFORO NO LIQUIDO CEFALORRAQUEANO DURANTE CRISES CONVULSIVAS
}

\author{
A. Spina-França * \\ Luís M. DE Assis * \\ J. M. MARLET ***
}

\author{
WALdemar Salvia ** \\ Francisco B. De Jorge* \\ MiltoN ZaIDAN **
}

Estudos recentes têm demonstrado que na epilepsia ocorrem distúrbios do metabolismo hidreletrolítico. Assim, foi verificado que há aumento da taxa de potássio no líquido extracelular ${ }^{2}$ durante crises convulsivas. Esta alteração, demonstrada em vários tecidos, ocorre também no cérebro, sugerindo aumento da permeabilidade das membranas celulares do parênquima cerebral durante as crises 13,14 .

Já foi demonstrada a importância dos íons cloro e sódio na hiperexcitabilidade convulsivante ${ }^{6,15}$. Em crises convulsivas induzidas por $\mathrm{CO}_{2}$ ocorre rápido influxo de sódio para o espaço intracelular ${ }^{16,17}$. Parece que a sensibilidade a crises convulsivas varia no sentido inverso da relação entre sódio extra e intracelular ${ }^{8}$.

Alteraçōes metabólicas de compostos de fósforo de alta energia foram verificadas no cérebro de epilépticos por ocasião de crises convulsivas. Têm sido registradas paralelamente alterações do fósforo iônico em convulsões $4,14,15$.

Os estudos referidos foram feitos em amostras de tecido cerebral de sêres humanos ou de animais e em amostras de liquido extracelular intravascular ${ }^{1}$. É admissivel que os dados obtidos pelo exame do sangue representam, pelo menos em parte, o resultado da atividade metabólica muscular desencadeada durante a crise, conforme sugere o fato de não ocorrerem modificações da composição iônica do sangue em crises de pequeno mal ${ }^{11}$. As modificações determinadas pela atividade anormal das células nervosas devem refletir-se de modo mais imediato na composição dos liquidos extravasculares que entram em contato com elas, isto é, pelos liquidos intersticial e cefalorraqueano ${ }^{10}$. No que se refere ao líquido intersticial cerebral, os estudos têm sido feitos em geral em animais de laboratório ${ }^{7}$. A intimidade das relações entre o tecido nervoso e o líquido cefalorraqueano (LCR) permite que a composição dêste último represente meio dos mais adequados para êsses estudos no homem. Assim sendo, foi estudado o comportamento

Trabalho da Clínica Neurológica (Prof. A. Tolosa), da Clinica Médica (Prof. A. B. Ulhoa Cintra) e da Clínica Pediátrica (Prof. Pedro de Alcântara M. Machado) da Faculdade de Med. da Univ. de São Paulo: *Assistente; ** Médico plantonista de Pronto Socorro; *** Acadêmico estagiário. 
do ionograma do LCR de pacientes na vigência de crises convulsivas, no sentido de verificar as alterações provocadas pela atividade celular anormal.

\section{MATERIAL E MÉTODOS}

As concentrações de cloro, sódio, potássio e fósforo inorgânico foram determinadias em amostras de LCR colhidas de pacientes na vigência ou imediatamente após crises convulsivas (grupo I). O grupo compreende 33 crianças: em 9 a crise convulsiva não era acompanhada de outra sintomatologia (casos de 1 a 9 ; subgrupo I $a$ ); em 12 a convulsão ocorreu na vigência de hipertemia (casos de 10 a 21; subgrupo $\mathrm{I} b$ ); em 12 a convulsão ocorreu durante reidratação (casos de 22 a 33; subgrupo $\mathrm{I} c$ ).

Para fins comparativos foram reunidos outros dois grupos de casos (grupos II e III). O grupo II compreende 17 pacientes com epilepsia generalizada, sendo o LCR colhido em periodos intercríticos: tratava-se de 11 adultos (casos de 34 a 44) e 6 crianças (casos de 45 a 50). O grupo IIr é representado por 25 pacientes portadores de afecções neurológicas crônicas, sem modificações apreciáveis do estado geral e sem sintomatologia convulsiva; o grupo consta de 18 adultos e 7 crianças. Éste grupo foi tomado como referência à normalidade e os dados referentes a êle constituíram motivo de estudo de outra publicação mais particularizada sôbre o assunto ${ }^{1 ?}$.

As amostras de LCR submetidas a exame não apresentavam alterações no aspecto, côr, citologia e concentracão protêica; os resultados das reações de Pandy e de fixação do complemento para sifilis e para cisticercose foram negativos.

A concentração do cloro foi determinada pelo método de Schales e Schales" a de fósforo inorgânico pelo de Fiske e Subbarow ${ }^{5}$ e as de potássio e sódio por fotometria de chama. Os resultados são expressos em $\mathrm{mg} / 100 \mathrm{ml}$ para a fosforraquia e em $\mathrm{mEq} / \mathrm{l}$ para os demais componentes estudados*.

\section{RESULTADOS}

Os resultados obtidos para os 50 casos dos grupos I e II são apresentados no quadro 1.

No quadro 2 são apresentadas as médias referentes a cada um dos componentes estudados nos três grupos de casos. No gráfico 1 é ilustrado o comportamento das taxas verificadas para o grupo I em relação às estimativas do grupo III.

1 - Pacientes portadores de afecçóes neurológicas crônicas, não acompanhadas de sintomatologia convulsiva (Grupo III) — Os valôres médios representativos das concentraçōes dos componentes do LCR estudados se encontram dentro dos limites apontados como normais na literatura, conforme foi demonstrado na publicaçāo anterior já citada ${ }^{12}$. As diferenças existentes entre os valôres das concentrações encontradas para adultos e para crianças incluidas neste grupo não se mostraram significativas ( $t=1,2$ para cloro; 1,5 para sódio; 0,15 para potássio e 1,8 para fósforo). Os valôres representativos dêste grupo de casos foram tomados como têrmo de comparação para os outros dois grupos apresentados nesta publicação.

2 - Pacientes com epilepsia cujo LCR foi examinado no intervalo entre crises convulsivas (Grupo II) - Em relação aos resultados obtidos para o grupo anterior não foram encontradas modificações significativas da composição do LCR dêstes casos. Os valôres de $t$ obtidos foram os seguintes: adultos 0,7 para cloro; 1,9 para

* Agradecemos aos técnicos Sr. Antonio Germano da Silva e Sra. Lilia A. de Vilhena Nunes a colaboração prestada nas análises químicas. 


\begin{tabular}{|c|c|c|c|c|c|c|c|c|c|}
\hline Caso & $C l$ & $N a$ & $K$ & $P$ & Caso & $C l$ & $N a$ & $K$ & $P$ \\
\hline 1 & 129 & 163 & 3,50 & 2,22 & 26 & 100 & 138 & 3,81 & 2,68 \\
\hline 2 & 135 & 166 & 4,12 & 1,68 & 27 & 99 & 138 & 4,20 & 2,52 \\
\hline 3 & 123 & 157 & 3,97 & 2,22 & 28 & 102 & 142 & 4,00 & 1,90 \\
\hline 4 & 132 & 150 & 3,55 & 1,66 & 29 & 103 & 139 & 3,80 & 1,94 \\
\hline 5 & 121 & 159 & 3,10 & 2,42 & 30 & 102 & 141 & 3,30 & 2,88 \\
\hline 6 & 134 & 157 & 3,10 & 1,38 & 31 & 101 & 139 & 3,80 & 2,60 \\
\hline 7 & 132 & 150 & 4,70 & 3,75 & 32 & $\$ 9$ & 138 & 3,90 & 2,52 \\
\hline 8 & 129 & 160 & 3,48 & 1,78 & 33 & 100 & 142 & 4,00 & 4,02 \\
\hline 9 & 129 & 164 & 3,42 & 2,20 & 34 & 134 & 148 & 2,95 & 0,88 \\
\hline 10 & 128 & 150 & 3,00 & 1,36 & 35 & 127 & 146 & 3,15 & 1,10 \\
\hline 11 & 134 & 156 & 3,61 & 2,08 & 36 & 130 & 150 & 3,00 & 1,08 \\
\hline 12 & 124 & 153 & 3,72 & 1,94 & 37 & 128 & 150 & 3,50 & 0,86 \\
\hline 13 & 129 & 164 & 5,37 & 2,08 & 38 & 127 & 153 & 3,00 & 1,10 \\
\hline 14 & 133 & 159 & 4,00 & 3,12 & 39 & 130 & 148 & 2,90 & 0,82 \\
\hline 15 & 131 & 150 & 2,90 & 1,32 & 40 & 127 & 152 & 2,95 & 1,40 \\
\hline 16 & 130 & 168 & 3,70 & 1,92 & 41 & 124 & 135 & 3,00 & 1,42 \\
\hline 17 & 129 & 166 & 3,40 & 2,10 & 42 & 125 & 139 & 2,80 & 1,61 \\
\hline 18 & 134 & 153 & 3,40 & 1,88 & 43 & 119 & 137 & 2,80 & 1,55 \\
\hline 19 & 129 & 159 & 3,91 & 2,08 & 44 & 125 & 140 & 2,80 & 1,42 \\
\hline 20 & 130 & 128 & 3,60 & 1,42 & 45 & 133 & 144 & 2,80 & 1,32 \\
\hline 21 & 126 & 159 & 3,51 & 1,70 & 46 & 138 & 157 & 3,35 & 1,32 \\
\hline 22 & 120 & 137 & 3,32 & 1,98 & 47 & 135 & 150 & 2,85 & 1,24 \\
\hline 23 & 104 & 124 & 4,30 & 1,56 & 48 & 132 & 145 & 2,90 & 1,46 \\
\hline 24 & 129 & 157 & 3,80 & 2,60 & 49 & 125 & 137 & 3,10 & 1,29 \\
\hline 25 & 133 & 152 & 4,70 & 3,64 & 50 & 123 & 139 & 2,90 & 1,50 \\
\hline
\end{tabular}

Quadro 1 - Resultados das determinações das taxas de cloro, sódio, potássio e fósforo inorgânico no LCR de 50 pacientes epilépticos; nos casos de 1 a 33 o LCR foi colhido durante ou imediatamente após crise convulsiva; nos casos de 34 a 50 o LCR foi colhido em periodo intercrítico. Legenda: taxas de cloro, sódio e potássio em $\mathrm{mEq} / \mathrm{l}$; taxas de fósforo em $\mathrm{mg} / 100 \mathrm{ml}$.

\begin{tabular}{ccccc}
\hline Grupo & $C l$ & $N a$ & $K$ & $P$ \\
\hline I $a$ & 129 & 158 & 3,66 & 2,15 \\
I $b$ & 139 & 155 & 3,68 & 1,92 \\
Ic & 108 & 141 & 3,91 & 2,57 \\
\hline II & 128 & 145 & 2,99 & 1,26 \\
\hline III & 128 & 150 & 3,04 & 1,27 \\
\hline
\end{tabular}

Quadro 2 - Médias representativas do comportamento dos componentes inorgánicos estudados no LCR, segundo os grupos de casos. 
sódio; 0,6 para potássio e 0,8 para fósforo; erianças: 1,1 para cloro, 1,4 para sódio, 0,7 para potássio e 1,1 para fósforo. Não foram encontradas diferenças em relação aos valôres-contrôle, portanto, na concentração de cloro, de sódio, de potássio e de fósforo no LCR de pacientes epilépticos em que a determinação foi feita no intervalo de crises convulsivas.

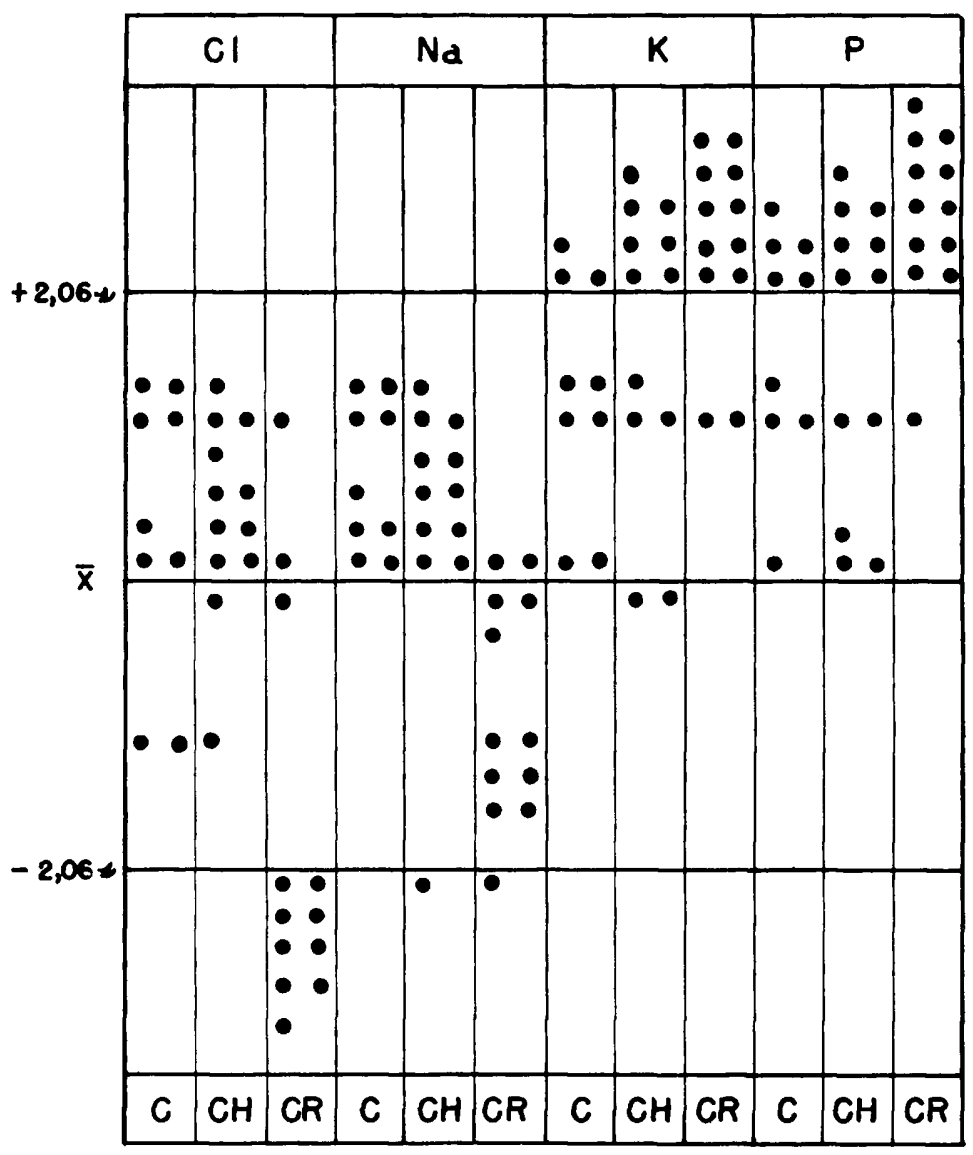

Gráfico 1 - Comportamento das taxas de cloro, sódio, potássio e fósforo nas amostras de LCR colhidas na vigência de crise convulsiva (grupo I) em relação aos parâmetros do grupo contrôle (grupo III). Legenda: $x$, média; s, desvio padrão; $C$, convulsão não associada a outro sintoma; $C H$, convulsão durante hipertermia; $C R$, convulsĩo durante reidratas̆ão.

3 - Pacientes cujo LCR foi examinado na vigência de crises convulsivas (Grupo I) - Em relação às estimativas do grupo normal foram encontradas alteraçōes dos componentes inorgânicos do LCR estudados neste grupo de casos (gráfico 1). $\mathrm{Em} 20$ casos foi encontrado aumento da concentração de potássio e em 23 da de fósforo. A concentração de sódio estava diminuida em 2 casos e a de cloro, em 9. A diminuição da concentração de cloro só foi observada entre as crianças em que a convulsão ocorreu durante a reidratação (subgrupo Ic). 
O comportamento dos componentes inorgânicos estudados no LCR no grupo III diferiu daquele verificado nas crianças dos grupos I e II. As diferenças encontradas foram analisadas estatisticamente (teste de Kruskal-Wallis) e se mostraram altamente significativas $\left(\chi^{2}=18,63\right.$ para cloro; 18,50 para sódio; 22,76 para potássio e 25,13 para fósforo).

Pela análise do gráfico 1 verifica-se que essas diferenças não ocorreram de modo homogêneo em relação aos três tipos de pacientes com convulsões analisados no grupo I. As diferenças podem ser expressas pelas médias referentes a cada um dos tipos de casos (quadro 2). Verifica-se que havia aumento das taxas de potássio e de fósforo nos três subgrupos de casos; os aumentos encontrados foram estatisticamente significativos. Para o potássio os valôres de $t$ foram: 3,4 nos pacientes do subgrupo Ia; 3,4 nos do subgrupo Ib; 7,2 nos do subgrupo Ic. Para o fósforo os valôres de $t$ foram, respectivamente, $3,7,4,3$ e 6,2 .

Nas crianças com crise convulsiva na vigência de hipertermia (subgrupo $I b$ ) o comportamento das taxas de cloro e de sódio não diferia daquele verificado no grupo III ( $t=1,7$ para cloro e 1,4 para sódio).

Entre as crianças com crise convulsiva não associada a outra sintomatologia (subgrupo $\mathrm{I} a$ ), o comportamento do cloro não diferia do encontrado para os casos do grupo III $(t=0,56)$, enquanto que o do sódio era diferente $(t=2,96)$. A diferença se caracterizou pela ocorrência de taxas mais elevadas de sódio neste grupo de crianças com convulsão.

Entre as crianças em que a crise convulsiva ocorreu durante a reidratação (subgrupo $I c$ ) as taxas de cloro e de sódio no LCR apresentavam comportamento diverso daquele observado para o grupo III. As diferenças eram estatisticamente significantes ( $t=5,5$ para cloro e 3,0 para sódio) e se caracterizavam pela ocorrência de taxas mais baixas de cloro e de sódio no LCR das crianças com convulsão.

No grupo III (contrôle de normalidade) foi verificado que não havia dependência entre as variações das taxas de potássio e de fósforo $(r=-0,03)$. O mesmo fato foi verificado entre os pacientes do grupo II $(r=-0,15)$. Para os 33 pacientes do grupo I, era significativa a correlação entre as variações das taxas dêsses componentes no LCR $(r=+0,46)$. Esse dado indica que há dependência entre os aumentos das taxas de potássio e de fósforo inorgânico no LCR na vigência de crise convulsiva. A dependência verificada é direta, isto é, à medida em que aumenta a concentração de um dêsses componentes tende a aumentar também a do outro.

\section{COMENTARIOS}

No intervalo de crises convulsivas não foram encontradas no LCR alterações da concentração dos íons estudados.

$E$ fato estabelecido que as crises convulsivas resultam de despolarização das membranas de neurônios cerebrais; durante a despolarização há entrada de sódio para as células e saída de potássio. Tower ${ }^{14}$ verificou aumento da concentração do sódio intracelular e queda da concentração do potássio intracelular em porções de cérebro de epiléticos.

Segundo essas experiências, ao se recompor o metabolismo celular após a descarga, êste último íon não é reabsorvido do meio, ao contrário do que acontece quando se observa o comportamento de porções de cérebro normal.

Esses dados, entre outros, permitem explicar o aumento da concentração de potássio no LCR na vigência de crises convulsivas, conforme foi observado para o material reunido nesta publicação. 
Há aumento de fósforo inorgânico no sangue venoso cerebral e possìvelmente no LCR também, por perda intracelular, ao desencadear-se crise convulsiva, conforme refere McIlwain ${ }^{3}$. $\mathrm{Na}$ série de casos apresentados foi possivel demonstrar o aumento da concentração de fósforo inorgânico no LCR e que êste se correlacionava de modo significativo com o aumento verificado para as concentrações de potássio.

O aumento dêsses íons na vigência de convulsão representariam a repercussão para o lado do LCR de alterações iônicas envolvidas no fenômeno de despolarização de membrana.

Os dados referentes ao ion sódio variaram conforme o subgrupo de pacientes com crise convulsiva. Esses dados sugerem que na entrada do sódio para a célula por ocasião dos fenômenos de despolarização da membrana o LCR não participa de modo direto. As variações nas taxas de sódio encontradas no LCR dos casos estudados (grupo I) poderiam decorrer de modificaçōes do próprio estado geral que intercorreram de modo diverso em cada um dos subgrupos. Confirma êsse ponto de vista o fato de as taxas diminuidas de sódio terem sido encontradas ao lado da diminuição das taxas de cloro no subgrupo Ic. Alterações do metabolismo iônico podem resultar de reidratação excessiva.

Millichap e col. ${ }^{9}$ verificaram hiponatremia, após convulsōes febris, em $24 \%$ dos casos estudados; explicaram êsse achado, em um dos casos, por hiperidratação ${ }^{7}$. Nos casos restantes foram encontrados, no sôro, valôres próximos do normal, concordando com os nossos achados em relação ao LCR, nos casos de convulsão com hipertermia.

\section{RESUMO E CONCLUSOES}

Estudo do comportamento das concentrações de cloro, sódio, potássio e fósforo inorgânico no LCR de 33 pacientes na vigência de crises convulsivas e de 17 pacientes epilépticos na interfase de crises convulsivas. Os resultados obtidos foram estudados em comparação aos valôres normais anteriormente relatados.

Não foram encontradas modificações quanto às taxas de íons estudados no LCR dos pacientes epilépticos examinados no período intercrítico.

Foram verificadas alteraçōes das taxas de ions estudados no LCR entre os pacientes estudados na vigência de crise convulsiva. As alterações encontradas se caracterizavam por aumento das taxas de potássio e de fósforo inorgânico. Os aumentos encontrados foram estatisticamente significantes. A taxa de sódio no LCR dos pacientes cuja crise convulsiva não se associava a outras manifestações de doença se apresentava, em média, aumentada. Diminuição das taxas médias de sódio e de cloro foi constatada entre os pacientes cuja crise convulsiva ocorreu durante reidratação.

Os resultados foram discutidos em relação a dados da literatura quanto às modificações do metabolismo iônico envolvidas nos fenômenos de despo- 
larização de membrana, que ocorrem na crise convulsiva. Os aumentos verificados para as concentrações de potássio e de fósforo inorgânico no LCR durante a crise convulsiva confirmam os dados quanto à passagem dêsses ions do espaço intracelular para o extracelular nessas condições, já registrados através de estudos feitos no sangue e em amostras de cérebro de epilépticos.

\section{SUMMARY}

Concentrations of $\mathrm{Cl}, \mathrm{Na}, \mathrm{K}$ and inorganic $P$ in the cerebrospinal fluid during convulsive seizures.

The concentrations of $\mathrm{Cl}, \mathrm{Na}, \mathrm{K}$ and inorganic $\mathrm{P}$ were determined in CSF samples of 50 patients with epilepsy. In 33 patients the sample studied was collected at the occasion of a convulsive seizure (group I). In 17 patients it was collected in the intermediate period between seizures (group II).

The patients of group I were children. They were grouped according to conditions associated to the convulsive seizure. In 9 , there were no other manifestation of disease (sub-group $\mathrm{I} a$ ). In 12 , the seizure was associated to hyperthermy (sub-group $\mathrm{I} b$ ) and in 12 it occurred during rehydration therapy (sub-group Ic).

The results were compared statistically to those obtained for a series of 25 non epileptic patients taken as control (group III). The results obtained for the latter fall in the normal range and were reported previously.

Results obtained for the patients of the group II did not differ from those found in the control group.

The concentrations of $\mathrm{K}$ and inorganic $\mathrm{P}$ were found to be increased in group I. Their mean differ significantly of those found for control group. There occurred a positive correlation between the concentrations of $\mathrm{K}$ and $\mathrm{P}$ in group $\mathrm{I}$. The value of the correlation was significant.

The findings regarding to the concentration of $\mathrm{Na}$ show shiftings that were not uniform. Their mean value was elevated in the sub-group Ia, normal in the sub-group $\mathrm{I} b$ and low in the sub-group Ic. The mean value of ine $\mathrm{Cl}$ concentration was low in the sub-group Ic. It was normal in the other two sub-groups.

Data concerning to the changes in the concentrations of $K$ and inorganic $\mathrm{P}$ in the CSF during convulsive seizures were discussed. Bioelectric changes of the cellular membrane during seizures are reported in the literature. They induce the passage of these components through the cellular membrane to the extracellular space thus resulting the elevation of their concentration in this space. The results presented make it possible to evaluate the extension of such changes in the fluid of the extravascular space. The latter is represented in the case by the CSF. 
The exchange of intracellular $K$ and inorganic $P$ to the extracellular space is accompanied by exchange of $\mathrm{Na}$ in the opposite direction. The $\mathrm{Na}$ concentration is referred to elevated in the intracellular space in these circumstances. The results reported suggest that $\mathrm{Na}$ is not provided by $\mathrm{CSF}$ only, for this exchange. Other compartments of the extracellular space and/or of the intracellular space may be more directly involved. This is suggested because the concentrations of $\mathrm{Na}$ in the CSF did not show uniform changes in the three sub-groups of patients with seizures that were considered. General changes in the extracellular fluid composition might explain the differences in the concentration of $\mathrm{Na}$ which were reported. The concentrations of $\mathrm{Na}$ and $\mathrm{Cl}$ found in the CSF of patients with convulsion during rehydration therapy are elucidative in this way. They were found to be low, suggesting an excess in the water uptake.

\section{REFERENCIAS}

1. COILRAUT, R.; JeANNETON, C. - Epilepsie et Métabolisme Céllulaire. Orientations Thérapeutiques. Libr. Maloine, Paris, 1959. 2. COLFER, H. F.; ESSEX, H. E. - The distribution of total electrolyte, potassium and sodium, in the cerebral cortex in relation to experimental convultions. Am. J. Physiol., 150:27-36, 1947. 3. ILWAIN, H. Mc - Biochemistry and the Central Nervous System. J. \& A. Churchill Ltd., London, 1955, pág. 233. 4. KLEIN, J. R.; OLSEN, N. S. - Effect of convulsive activity upon the concentration of brain glucose, glycogen, lactate and phosphats. J. Biol. Chem., 167:747-756, 1947. 5. KOLMER, J. A.; SPAULDING, E. H.; ROBINSON, H. W. - Approved Laboratory Technic, $5^{\natural}$ ed., Appleton, Century, Crofts Inc., New York, 1951. 6. MEYER, J. S.; GOTOH, F.; TAZAKI, Y. - Inhibitory action of carbon dioxid and acetazoleamide in seizure activity. EEG and Cl. Neurophysiol., 5:762-775, 1961. 7. MILLICHAP, J. G. - Studies on febrile seizures; the balance of water and electrolytes. Neurology 10:312-321, 1960. 8. MILLICHAP, J. G.; BALTER, M.; HERNANDEZ, P. - Development of susceptibility to seizures in young animals: brain water, electrolyte and acid-base metabolism. Proc. Soc. Exper. Biol. Med., 99:6-11, 1960. 9. MILLICHAP, J. G.; MADSEN, J. A.; ALEDORT, L. M. - Studies in febrile seizures: clinical and electroencephalographic study in unselected patients. Neurology 10:643-653, 1960. 10. MUSSA, G. C. - Valutazlone del liquido extracellulare nel lattante distrofico. Minerva Ped., 13:427-431, 1961.11. POCH, G. F. - Las modificaciones electroencefalográficas y electrolíticas determinadas por la inyección endovenosa de acetazolamida en enfermos con pequeño mal. Rev. Neurol. de Buenos Aires 19:31-35, 1961. 12. SPINA-FRANÇA, A.; De JORGE, F. B. - Concentrações de cloro, sódio, potássio e fósforo no liquido cefalorraqueano normal. Arq. Neuro-Psiquiat., 21:96-104, 1963. 13. TOWER, D. B. - Nature and extent of the biochemical lesion in human epileptogenic cerebral cortex. Neurology 5:113-130, 1955. 14. TOWER, D. B. - Neurochemistry of Epilepsy. Illinois, Springfield, Charles C. Thomas, 1960. 15. VAN HARREVELD, A.; ACHADE, J. P. - Changes in the electrical conductivity of cerebral cortex during seizure activity. Exp. Neurol., 5:383-400, 1962. 16. WOODBURY, D. M. - Redistribution of electrolytes (K, Na, P) following electroshock convulsions in rat. Am. J. Physiol., 155:479, 1948. 17. WOODBURY, D. M.; KOCH, A.; VERNADAKIS, A. - Relation between excitability and metabolism in brain as elucidated by anticonvulsant drugs. Neurology 8 (supl. 1):113-116, 1958.

Clínica Neurológica - Faculdade de Medicina da USP - Caixa Postal $\$ 461-$ São Paulo, SP - Brasil. 